\title{
Coding of Microsaccades in Three-Dimensional Space by Premotor Saccadic Neurons
}

\author{
Marion R. Van Horn and Kathleen E. Cullen \\ McGill University, Department of Physiology, Montreal, Quebec, H3G 1Y6 Canada
}

Microsaccades are small, involuntary eye movements that are produced during fixation. While accurate visual perception requires precise binocular coordination during fixation, previous studies of the neural control of microsaccades measured the movement of one eye only. Here we show how premotor saccadic neurons control these small fixational eye movements in three-dimensional space. Microsaccadic eye movements, produced by monkeys trained to fixate targets presented at different depths, were similarly distributed in three-dimensional space during both near and far viewing. Single unit recordings of the neural activity of premotor neurons further revealed that the brainstem saccadic circuitry controls these minute disconjugate shifts of gaze by preferentially encoding the dynamic movement of an individual eye (i.e., integrated control of conjugate and vergence motion). These findings challenge the traditional notion that microsaccades are strictly conjugate and have important implications for studies that use microsaccades to evaluate visual and attentional processing, as well as certain neurological disorders.

\section{Introduction}

When exploring a visual scene, we make saccadic eye movements that are interspersed with periods of fixation. Interestingly, even when our gaze is fixed, our eyes are not completely still. Although they were once thought to play no role in visual perception (Kowler and Steinman, 1979), recent work suggests that these small fixational eye movements serve many important functional roles. For example, microsaccade propensity and direction have been shown to be highly informative about the allocation of attention (Hafed and Clark, 2002; Engbert and Kliegl, 2003; Betta and Turatto, 2006; Pastukhov and Braun, 2010). Accordingly, correlations between microsaccade generation and neural activity are currently used to investigate higher cognitive behaviors as well as the basis of certain neurological disorders (Engbert, 2006; Martinez-Conde, 2006; Martinez-Conde et al., 2009; OteroMillan et al., 2011b).

Why and how microsaccades are produced remain important questions. Previous studies suggest that the direction of microsaccades can be biased by attention but are, on average, corrective, such that the eyes remains on target (Cornsweet, 1956; Guerrasio et al., 2010; Hafed, 2011; Otero-Millan et al., 2011a). Recent single neuron and lesion experiments, coupled with computational modeling of neural activity in the superior colliculus (SC), have led to the proposal that during fixation, the instantaneous locus of activity on the rostral SC is a stochastic process

\footnotetext{
Received Oct. 5, 2011; revised Dec. 14, 2011; accepted Dec. 26, 2011.

Author contributions: M.R.V.H. and K.E.C. designed research; M.R.V.H. performed research; M.R.V.H. analyzed data; M.R.V.H. and K.E.C. wrote the paper.

This study was supported by the Natural Science and Engineering Research Council of Canada and the Fonds de la Recherche en Santé du Québec. We thank J. Carriot, M. Jamali, D. Mitchell, A. Schneider, and A. Dale for critically reading the manuscript and S. Nuara and W. Kucharski for excellent technical assistance.

Correspondence should be addressed to Dr. Kathleen E. Cullen, McIntyre Medical Research Building, Room 1219, 3655, Prom. Sir William Osler, Montreal, PQ, H3G 1 Y6 Canada. E-mail: kathleen.cullen@mcgill.ca.

DOI:10.1523/JNEUROSCI.5054-11.2012

Copyright $\odot 2012$ the authors $\quad 0270-6474 / 12 / 321974-07 \$ 15.00 / 0$
}

with zero mean (Hafed et al., 2009). As neuronal activity jitters about zero, a microsaccade is produced when activity is sufficiently deviated from zero.

The results of experiments in the last decade have also implicated the SC and its saccadic premotor circuitry in generating eye movements in three-dimensional space (i.e., integrated control of conjugate and vergence movement) (for review, see Cullen and Van Horn, 2011; for an alternative view, see Walton and Mays, 2003). Thus, we hypothesize that if this proposed model of microsaccade generation is correct, then jitter across the SC during fixation should generate both disconjugate and conjugate microsaccades. To test this, we measured the dynamic movement of each eye during fixation of near and far targets and quantified the conjugacy of microsaccades. Next, to better understand the neural circuitry involved in generating microsaccades, we recorded the neural activity of individual premotor saccadic neurons. Our results demonstrate that microsaccades are symmetrically generated in three-dimensional space, and that the brainstem saccadic circuitry controls these minute, dynamic shifts of gaze.

\section{Materials and Methods}

Surgical procedures and behavioral paradigms. Two female rhesus monkeys (Macaca mulatta) were prepared for extracellular recording under aseptic conditions as described previously (Van Horn et al., 2008). All procedures were approved by the McGill University Animal Care Committee and were in compliance with the guidelines of the Canadian Council on Animal Care.

Head-restrained monkeys were seated in a primate chair and were trained to fixate targets aligned along the midline for 1-3 s. A far target was illuminated by projecting a $\mathrm{HeNe}$ laser onto a cylindrical screen (approximately isovergent) located $55 \mathrm{~cm}$ away from the monkey's head $\left(<4^{\circ}\right.$ convergence). A near target was illuminated using a computer controlled light emitting diode located $8 \mathrm{~cm}$ from the monkey's head. The timing and location of target illumination, data acquisition, and on-line 
FAR

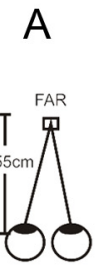

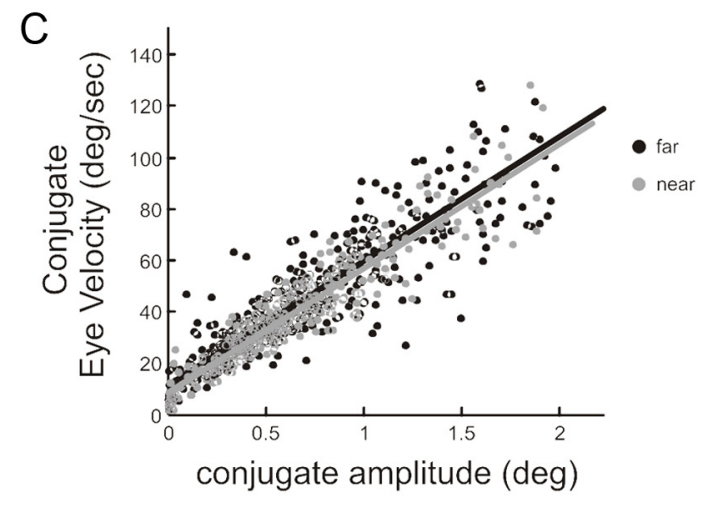

$\mathrm{E}$ conjugate
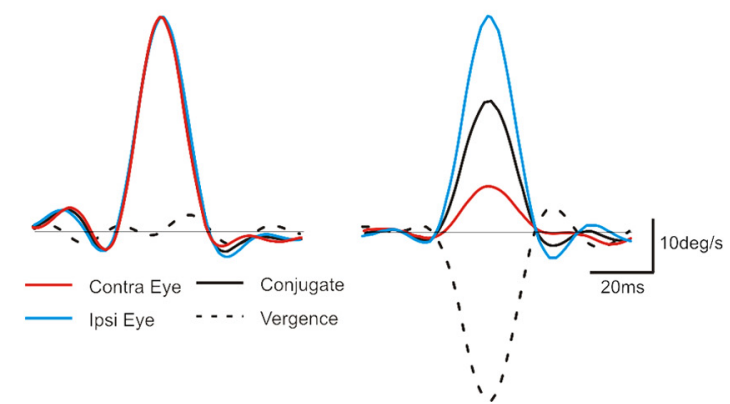

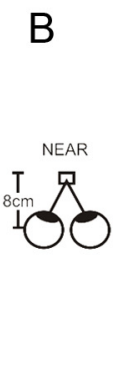

NEAR
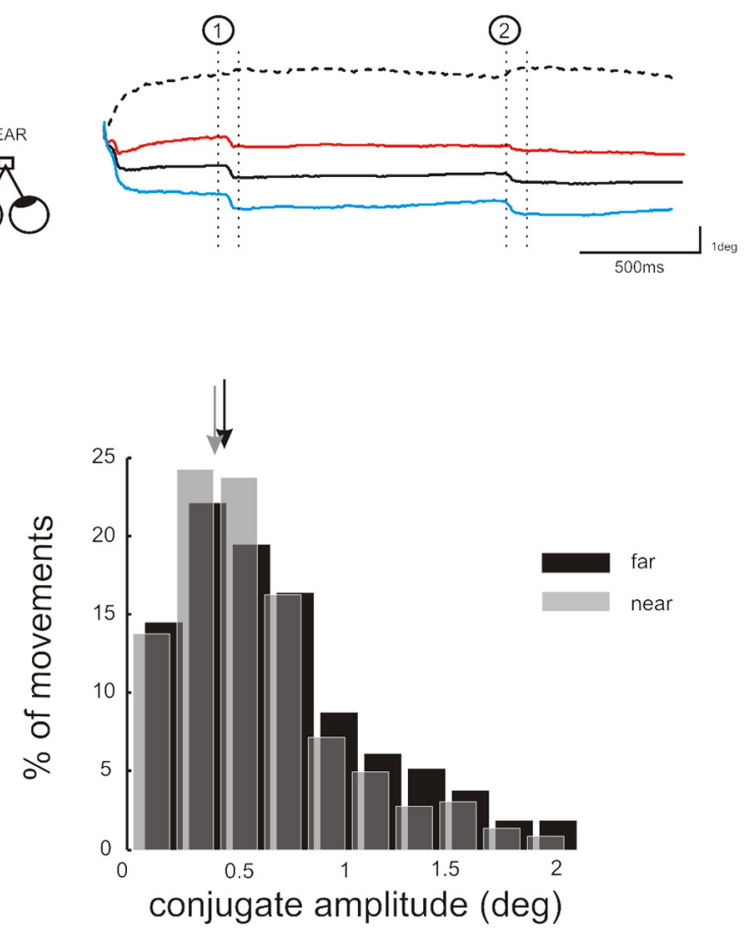

F
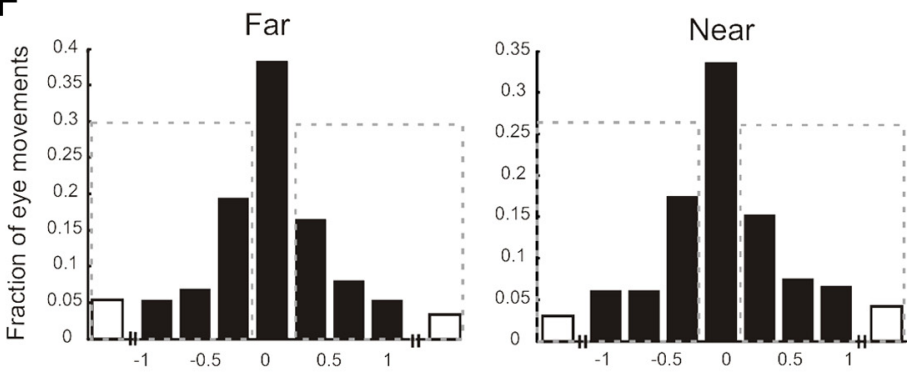

bino. index $=[$ peak vel left eye - right eye $] /$ [peak vel right eye + left eye $]$

Figure 1. $\quad \boldsymbol{A}, \boldsymbol{B}$, Example traces of the eye position during fixation of a far $(\boldsymbol{A})$ and near $(\boldsymbol{B})$ target. $\boldsymbol{C}$, Peak velocity-amplitude relationship for microsaccades during near ( $g$ ray dots; $N=365$ ) and far (black dots; $N=362$ ) fixation. $\boldsymbol{D}$, Distribution of the size of microsaccades detected during near (gray) and far (black) fixation. $\boldsymbol{E}$, Example velocity profiles of a conjugate (left) and disconjugate (right) microsaccade. $\boldsymbol{F}$, Distribution of the degree of conjugacy across all microsaccades during far and near viewing. Open bars denote movements where the eyes moved in opposite directions. Dashed gray boxes denote microsaccades where the movement of eye each differed by $>20 \%$. Contra, Contralateral; Ipsi, ipsilateral; bino., binocular; vel, velocity.

data displays were controlled using REX, a UNIX-based real-time acquisition system (Hays et al., 1982).

Single unit data acquisition and analysis. Extracellular single unit activity was recorded using tungsten microelectrodes (FHC). Saccadic burst neurons (SBNs; $N=25$ ), omnipause neurons (OPNs; $N=10$ ), and medial rectus motoneurons $(N=8)$ were identified on-line based on their stereotypical discharge properties during eye movements. Notably, since microsaccades often have a significant vertical component, we only analyzed SBNs that were completely silent during vertical saccades $(N=10)$.

The magnetic search coil technique was used to record the horizontal and vertical positions of both eyes (Judge et al., 1980). Each eye coil signal was calibrated independently by having the monkey fixate, with one eye masked, a variety of targets at different horizontal eccentricities and depths. Position signals were low-pass filtered at $250 \mathrm{~Hz}$ (analog 8 pole Bessel filter) and sampled at $1 \mathrm{kHz}$.

Data were sorted into periods of fixation where the monkey fixated either a far (convergence angle $<4^{\circ}$ ) or near (convergence $>4^{\circ}$ ) target for $>500 \mathrm{~ms}$. Microsaccades were detected using a $5 \mathrm{deg} / \mathrm{s}$ radial velocity criterion. Data were also visually sorted to verify that microsaccades were not erroneously missed or falsely detected (Hafed et al., 2009). Eye movements are reported in conjugate [conjugate $=($ left eye + right eye $) / 2$ ] and vergence (vergence $=$ left eye - right eye) coordinates.

In addition, to determine the proportion of disconjugate versus conjugate microsaccades, we calculated a binocularity index $\left(I_{\text {bino }}\right)$ as follows: $I_{\text {bino }}=\left[\max \left(\dot{E}_{l}\right)-\max \left(\dot{E}_{r}\right)\right] /\left[\max \left(\dot{E}_{l}\right)+\max \left(\dot{E}_{r}\right)\right]$, where $\dot{E}_{l}$ and $\dot{E}_{r}$ refer to the velocity of the left and right eye, respectively. In cases where the eyes moved in opposite directions, the movements $(\sim 8 \%$ of microsaccades) were considered independently.

The neuronal responses during saccades were characterized using linear optimization techniques (Cullen et al., 1996). First, firing rates were represented by a spike density function in which a Gaussian function ( $\mathrm{SD}=5 \mathrm{~ms}$ ) was convolved with the spike train. The specific model structures used are reported in Results, below. The goodnessof-fit of the data to each model was quantified using the VarianceAccounted-For $(\mathrm{VAF}=1-[\operatorname{var}(\operatorname{modFR}) / \operatorname{var}(\mathrm{FR})]$, where modFR represents the modeled firing rate and FR represents the actual firing 
A conjugate
SBN
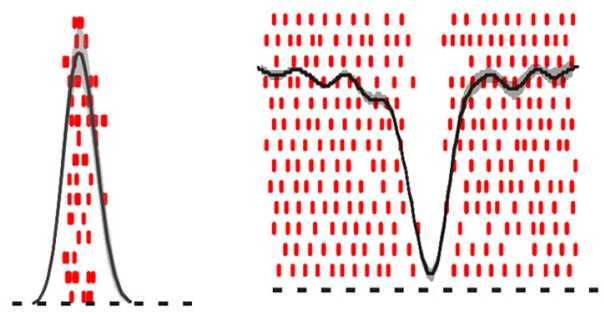

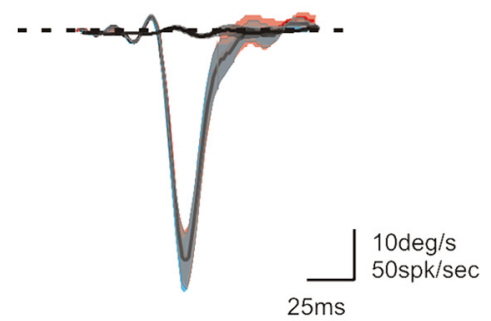

B
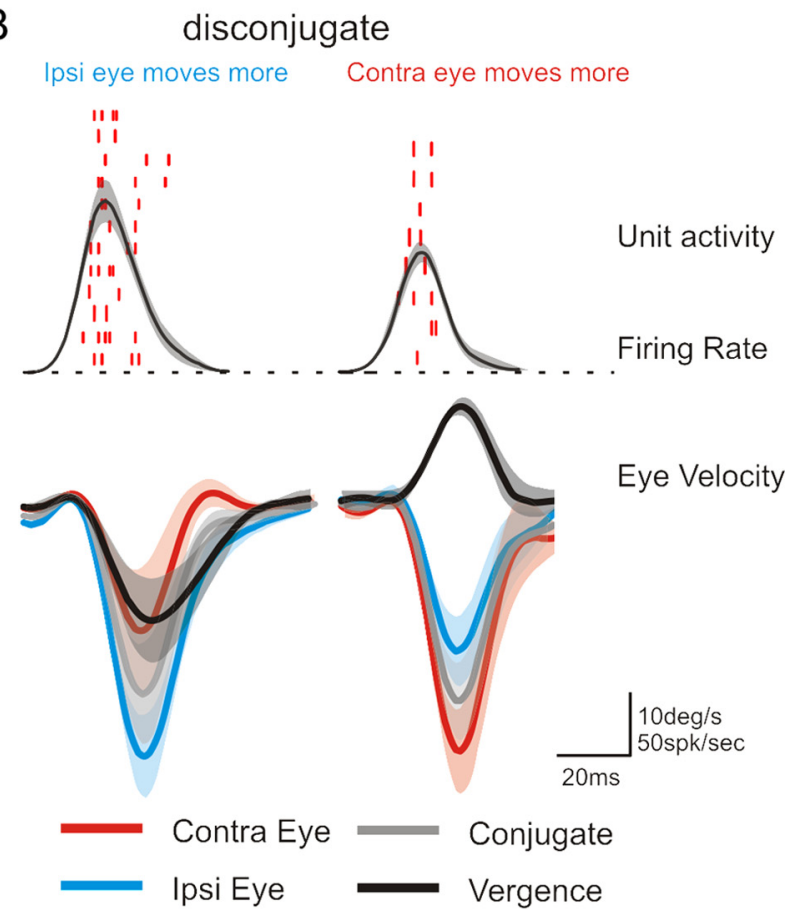

C

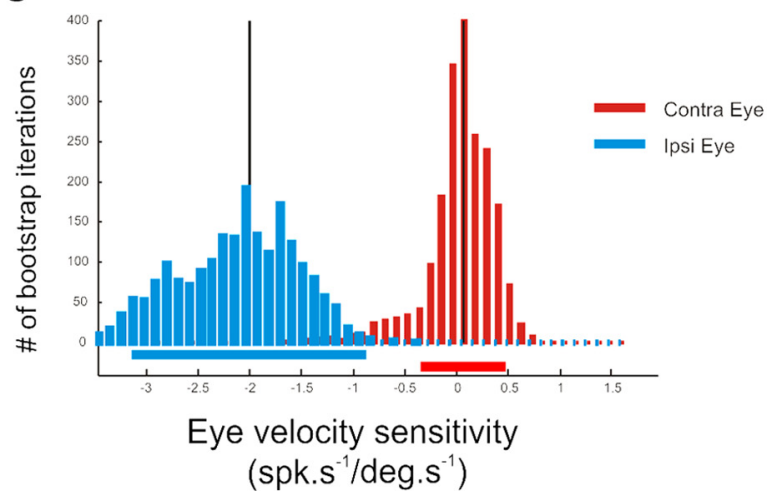

Figure 2. A, Example neural activity from a typical SBN (left) and OPN (right) during conjugate microsaccades aligned on saccade onset. Average firing rate with standard deviations is rate) (Cullen et al., 1996). For analysis of the neural control of disconjugate saccades, we considered movements for which one eye moved twice as much as the other eye in the on-direction of the neuron (Van Horn et al., 2008). In addition, we computed 95\% confidence intervals using a nonparametric bootstrap approach (Sokal and Rohlf, 1995; Carpenter and Bithell, 2000) to identify nonsignificant or identical model parameters (Sylvestre and Cullen, 2002). The Bayesian information criterion (BIC) (Schwartz, 1978), which served as a cost index, was calculated for each model estimation to determine whether removing the term was justified. For each neuron, to quantify the ocular preference (see Fig. 3), a Ratio index was defined as follows: $r=\min \left(r_{\mathrm{IE}}, r_{\mathrm{CE}}\right) / \max \left(r_{\mathrm{IE}}, r_{\mathrm{CE}}\right)$, where $r_{\mathrm{IE}}$ and $r_{\mathrm{CE}}$ are constants that represent the neuron's ipsilateral and contralateral velocity sensitivity, respectively.

Data are described as mean \pm SDs and a two-tailed, unpaired Student's $t$ test was used to determine whether the averages of two measured parameters were significantly different.

\section{Results}

In this study, we investigated the neural mechanisms underlying microsaccade generation during three-dimensional viewing. First, we evaluated the occurrence and conjugacy of microsaccades as a function of viewing depth. Second, we described the dynamic movement of each eye during microsaccades to determine whether microsaccades are produced throughout threedimensional space. Finally, we recorded the extracellular activity of premotor and motor neurons, which are known to dynamically encode the velocity of larger, voluntary saccadic eye movements, to determine their role in enabling the generation of conjugate and disconjugate microsaccades.

\section{Characterization of microsaccade dynamics during near versus far viewing}

We first compared eye movements during prolonged fixation of the far versus near target (Fig. $1 A, B$ ) and detected microsaccades in both viewing conditions. In agreement with previous studies, during far viewing, microsaccade amplitude was proportional to peak saccade velocity, consistent with the main sequence of larger saccades (far, $R=0.92$; Fig. $1 C$, black dots). Moreover, we found that during near viewing, saccade amplitude was also proportional to peak saccade velocity (near, $R=0.9$; Fig. $1 C$, gray dots,). A comparison of microsaccade amplitude revealed comparable distributions in the two conditions (mean far $=0.65^{\circ}$, mean near $=0.62^{\circ}, p>0.05$; Fig. $\left.1 D\right)$.

The analysis of each eye's temporal dynamics revealed that microsaccades are not strictly conjugate. While the movement of each eye was identical during some microsaccades (i.e., conjugate; Fig. $1 E$, left), we found that this was often not the case (i.e., disconjugate; Fig. $1 E$, right). To determine the proportion of disconjugate versus conjugate microsaccades in each viewing condition, we calculated a binocularity index (see Materials and Methods, above), for which a zero value indicated a conjugate movement, while values greater or less than zero indicated a disconjugate movement (i.e., a convergent or divergent movement, respectively). Overall, the distribution of indices were not significantly different $(p>0.05)$ and were centered on zero (mean far $=0.002 \pm 0.5$, mean near $=0.05 \pm 0.5)$. However, a signifi-

superimposed on the unit activity. Average ipsilateral (Ipsi), contralateral (Contra), conjugate, and vergence velocities traces are shown below the raster plots. $\boldsymbol{B}$, Example raster plots on the same example SBN shown in $\boldsymbol{A}$ during disconjugate saccades where the ipsilateral (left) or contralateral (right) eye moved more. C, Bootstrap histograms and 95\% confidence intervals (thick horizontal bars) for example SBN. 
A

\section{Saccadic Burst Neurons}

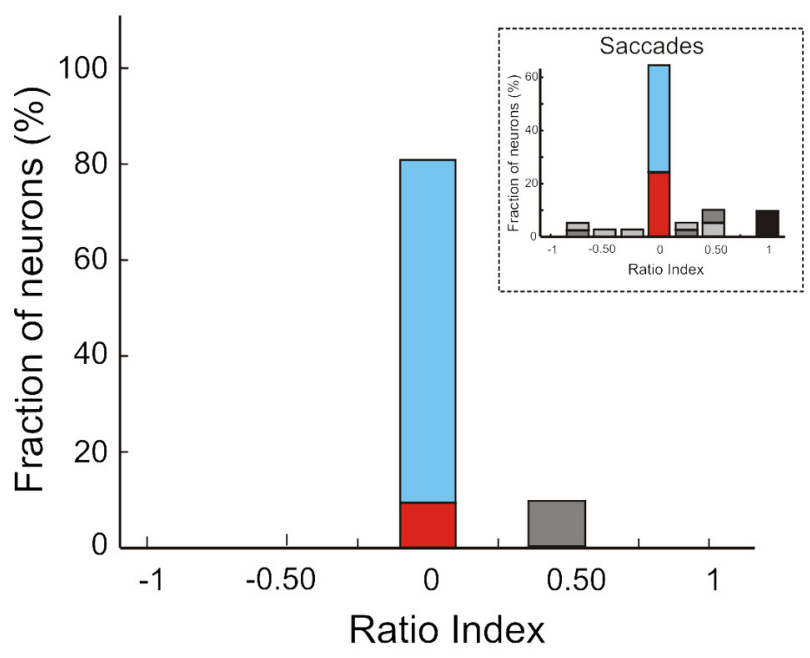

B

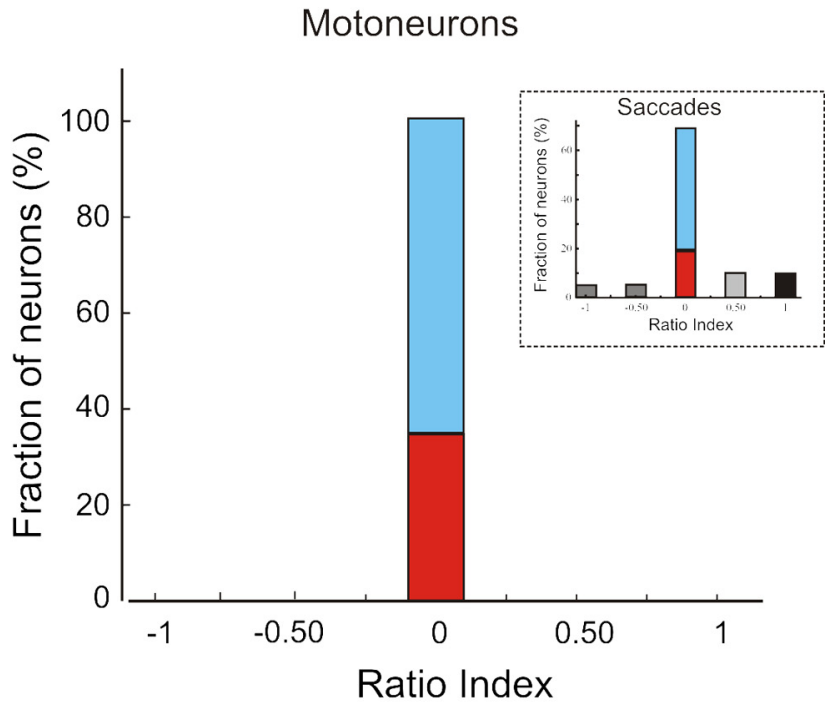

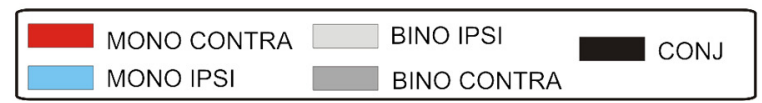

Figure 3. $\quad \boldsymbol{A}, \boldsymbol{B}$, Distribution of ratio indexes for SBNs $(\boldsymbol{A})$ and motoneurons ( $\boldsymbol{B})$ (see Materials and Methods). Insets show distributions of ratios for a sample of SBNs (left) and motoneurons (right) estimated in previous studies (Van Horn and Cullen, 2008). MONO, Monocular; BINO, binocular; CONTRA, contralateral; IPSI, ipsilateral; CONJ, conjugate.

cant proportion of the movements were characterized by nonzero values (Fig. $1 F$ ). In both conditions, one eye moved at least $20 \%$ more than the other in over half of the detected microsaccades (e.g., near $=56 \%$, far $=58 \%$; Fig. $1 F$, dashed boxes) and in a small percentage of the movements the eyes moved in opposite directions; this was comparable in both viewing conditions (near $=7 \%$, far $=8 \%$; Fig. $1 F$, open bars).

\section{Discharge characteristics of saccade-related neurons during microsaccades}

To understand how the brain controls disconjugate versus conjugate microsaccades, we recorded from SBNs and OPNs, which are known to be important players in generation of conjugate saccadic eye movements (Scudder et al., 2002). The discharge of a typical SBN and OPN is illustrated in Figure 2A. SBNs generated a vigorous burst of spiking activity just before the onset of ipsilateral, conjugate microsaccadic eye movements (Fig. 2A, left) and generated very few, if any, spikes during contralateral and vertical saccades (data not shown). In contrast, OPNs ceased firing throughout the duration of the microsaccade (Fig. $2 \mathrm{~A}$, right). To quantify these responses, SBN burst activity and OPN pause activity was first characterized using a classical metric-based analysis. As has been shown for larger saccades, we found that during conjugate microsaccades, the number of spikes in a SBNs burst was linearly related to the conjugate amplitude of the eye movement (population mean: $R=0.68 \pm 15$ ) and the pause duration of the OPN was linearly related to the duration of the microsaccade (population mean: $R=0.58 \pm 33$ ).

We next found that the same simple model, called the conjugate model, which describes the dynamic relationship between SBNs discharges and conjugate saccades (Cullen and Guitton, 1997), also well describes the relationship between SBNs discharges and conjugate microsaccades: $\operatorname{FR}(t)=b+r \dot{E}\left(t-t_{d}\right)$, where $\operatorname{FR}(t)$ is the estimated firing rate, $b$ is a bias, $r$ is a constant that represents the neuron's eye velocity sensitivity, $\dot{E}$ refers to the instantaneous veloc- ity of the eye, and $t_{d}$ is the neuron's dynamic lead time (VAF of $0.47 \pm 0.2$ across the population of SBNs). Next, we tested whether this same model could be used to predict SBN discharges during disconjugate microsaccades. Figure $2 B$ illustrates the discharge of the same example SBN during microsaccades where either the ipsilateral or contralateral eye moved more (Fig. $2 \mathrm{~B}$, left and right, respectively). Note that although the conjugate velocities were similar in the two conditions, the neuron's firing rate was strikingly different. Indeed, a conjugate-based model could not predict SBN activity; rather, neuronal responses were best described by an ipsilateral movement-based model $\left(\mathrm{VAF}_{\text {pred-cj }}=0.28\right.$ vs $\left.\mathrm{VAF}_{\text {pred-ipsi }}=0.46\right)$. On average, using ipsilateral eye velocity (rather than conjugate eye velocity) as $\dot{E}$ provided a $37 \%$ better prediction of the firing rate. This result was consistent across the population of the SBNs recorded in this study.

To further this, we asked whether a more complete binocular model might more accurately describe the firing rate of SBNs during disconjugate microsaccades: $\operatorname{FR}(t)=b+r_{\mathrm{IE}} \mathrm{E} \mathrm{E}\left(t-t_{\mathrm{d}}\right)+$ $r_{\mathrm{CE}} \dot{\mathrm{CE}}\left(t-t_{\mathrm{d}}\right)$, where $r_{\mathrm{IE}}$ and $r_{\mathrm{CE}}$ are constants that represent the neuron's ipsilateral and contralateral velocity sensitivity, respectively, and İE and ĊE refer to the instantaneous velocity of the ipsilateral and contralateral eye, respectively. We estimated the $95 \%$ confidence intervals on the parameter values $r_{\mathrm{IE}}$ and $r_{\mathrm{CE}}$ using a bootstrapping technique (see Materials and Methods, above). Figure $2 C$ shows the distributions of the estimates obtained using the bootstrapping approach. Notably, the confidence intervals do not overlap with each other and could therefore not be replaced with a single term proportional to conjugate eye velocity. In addition, the confidence interval for the sensitivity of the contralateral eye velocity was not statistically different from zero. Consistent with these findings, we found that the fit of a model reduced to include only the ipsilateral eye (i.e., $r_{\mathrm{CE}}=0$ ) was nearly identical to that of the full binocular model $\left(\Delta \operatorname{VAF}_{\text {(reduced }}-\right.$ binocular $\left.)=0.1 \% ; \Delta \mathrm{BIC}=0.1 \%\right)$. Accordingly, these results confirm that the neuron's discharge reliably en- 


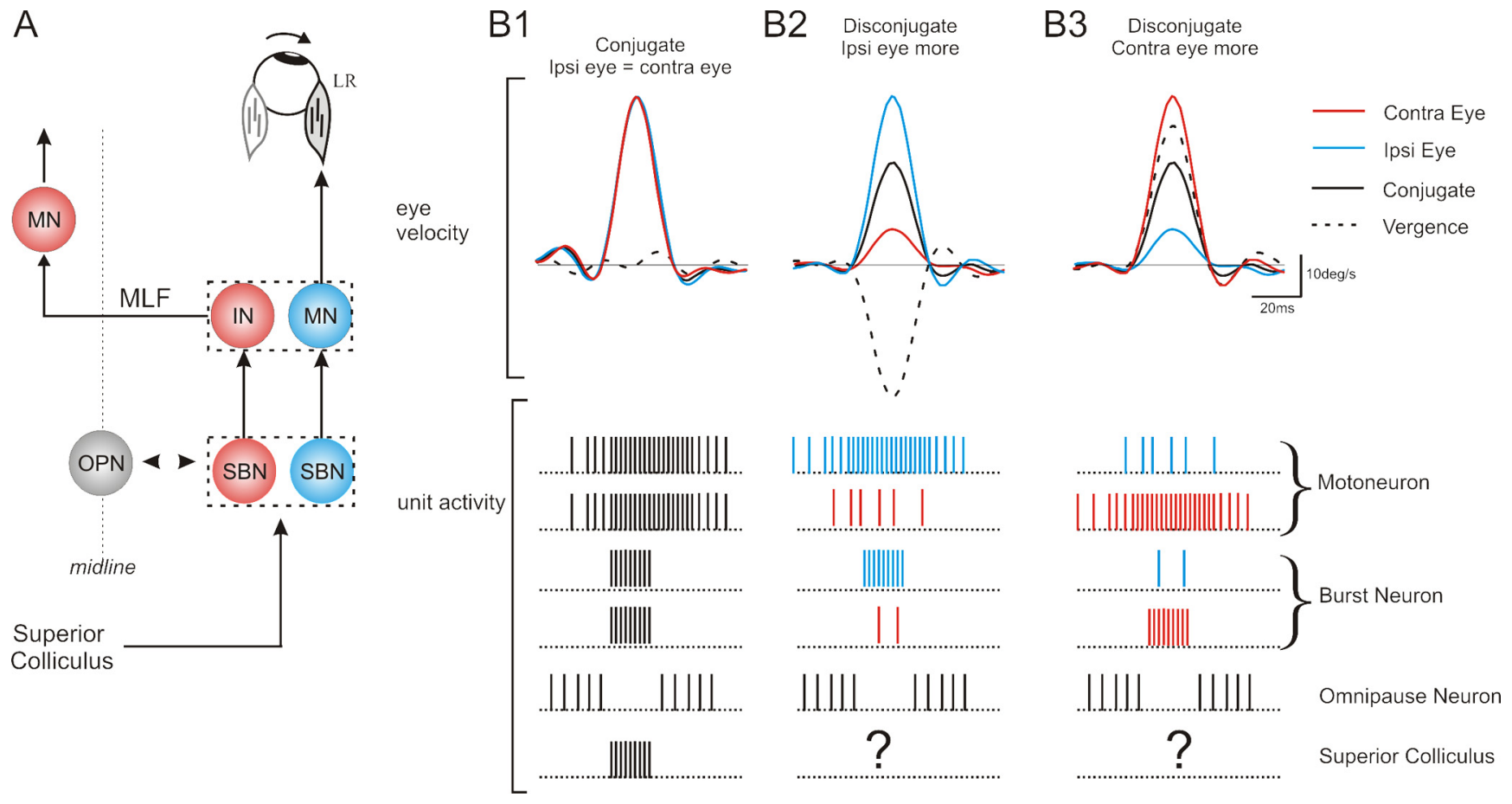

Figure 4. A, lllustration of the neural circuitry involved in generating microsaccades. The superior colliculus projects to the SBNs, which are reciprocally connected with the OPNs, and project directly to the motoneurons (MN) and internuclear neurons (IN) in the abducens, which project to the contralateral abducens via the medial longitudinal fasicularis (MLF). Neurons encoding the movement of the ipsilateral (Ipsi) and contralateral (Contra) eye are shown in blue and red, respectively. $\boldsymbol{B}$, Example unit activity of premotor neurons during example conjugate (B1) and disconjugate microsaccades $(\mathbf{B 2}, \mathbf{B} 3)$. Blue unit activity is the activity associated with a neuron related to the movement of the ipsilateral eye and red unit activity is representative of a neuron related to the movement of the contralateral eye. LR, Lateral rectus.

coded ipsilateral, but not contralateral or conjugate, eye motion during microsaccades.

Similar results were obtained for the majority of the neurons in our population of SBNs. Overall, $80 \%$ of our SBNs did not reliably encode contralateral eye motion during microsaccades (average $\Delta \mathrm{VAF}_{\text {(reduced vs binocular) }}=8.8 \pm 19.4 \% ; \Delta \mathrm{BIC}=1.1 \pm$ $3.3 \%)$, while a minority of the neurons were more accurately described using a contralateral eye or binocular model (Fig. $3 A$ ). We also performed a comparable analysis for a sample of extraocular motoneurons. Notably, a position term was also included in the model structure to account for the fact that motoneurons encode both eye position and velocity. As was the case for SBNs, we found that the majority of motoneurons (62.5\%) discharge reliably encoded ipsilateral, but not contralateral eye motion during microsaccades (Fig. 3B). Moreover, the 95\% confidence intervals for all of the neurons did not overlap with each other, confirming that individual eye velocity terms should not be replaced with a single conjugate term.

\section{Discussion}

In this study, we measured the dynamic movement of each eye during fixation and established that during microsaccades, the horizontal movement of one eye is not necessarily identical to the movement of the other (e.g., not conjugate). Notably, we found that microsaccades were distributed in three-dimensional space. Recordings from neurons in the saccadic pathway, specifically the premotor burst neurons of paramedian pontine reticular formation and their motoneurons, revealed that the brain controls these minute shifts of gaze in three dimensions by encoding the dynamic movement of an individual eye. Together, our results provide new insight into how the brain controls microsaccades, and in particular provide further evidence to contradict the tra- ditional view that the brain is circuited with independent pathways for conjugate and vergence control of saccades. Our finding that microsaccades are normally distributed in threedimensional space regardless of viewing distance has important implications for further models of the neural control of eye movements as well as for optimizing investigations of visual processing.

\section{Binocular microsaccades and the implication for studies of visual processing and visual awareness}

The results of numerous previous characterizations of the behavioral properties of microsaccades have suggested that they are, in effect, miniature versions of larger, voluntary saccades. For example, similar to larger saccades, the peak velocity of microsaccades is proportional to the amplitude of the corresponding movement. To make saccades between targets at optical infinity requires that both eyes rotate by the same angle (i.e., conjugate saccades). However, more often we look between objects located at different depths and thus the horizontal movement of each eye differs (i.e., disconjugate saccades). Surprisingly, the majority of studies examining microsaccades have assumed that these movements are strictly conjugate events. Here, we performed a complete analysis of the temporal dynamics of each eye during prolonged fixation of near and far targets and have established that microsaccades are not restricted to one plane of viewing but rather are distributed in three-dimensional space.

Overall, our findings have important implications for studies that use microsaccades to evaluate visual and attentional processing. There is accumulating evidence that the analysis of the timing, rate, and direction of microsaccades provide information about the neural mechanisms underlying vision and certain higher-order cognitive behaviors. For example, microsaccades, 
which modulate neural activity in visual areas (Martinez-Conde et al., 2000, 2002; Snodderly et al., 2001; Herrington et al., 2009; Hafed and Krauzlis, 2010; Hass and Horwitz, 2011), have also been shown to be correlated with visual awareness and visuospatial attention (Hafed and Clark, 2002; Engbert and Kliegl, 2003; Laubrock et al., 2005; Betta and Turatto, 2006; Engbert, 2006; Otero-Millan et al., 2008; Cui et al., 2009; Herrington et al., 2009; Kliegl et al., 2009; Pastukhov and Braun, 2010). Given that the temporal dynamics of each eye can differ throughout a given microsaccade, our results suggest that the knowledge of the motion of both eyes could improve the correlation observed between microsaccades and neural activity (or behavior). Moreover, evaluating the movement of the two eyes will give a more accurate estimation of the timing and frequency of microsaccade occurrence.

\section{A neurological bridge between microsaccades and saccades}

While the neural circuitry that underlies the generation of saccadic eye movements has been well characterized, a description of saccade-related neurons during fixational eye movements is virtually nonexistent. Recently, the rostral SC was found to have a causal role in the generation of microsaccades (Hafed et al., 2009). Since the SC is considered the primary excitatory input to the saccadic burst generator, it is likely that SBNs would similarly be involved in generating microsaccades. Only one previous study has reported that SBNs are responsive during very small amplitude saccades (Van Gisbergen et al., 1981) and this study was not specifically designed to study microsaccades but rather to provide a general model that would describe the neural activity of premotor neurons during saccades. Here, our quantitative analysis of the discharge dynamics revealed that the premotor saccadic burst neurons encode the size, duration, and velocity of microsaccades, and that a simple, first-order model provides an adequate description of the relationship between neural responses and eye motion. Furthermore, we extend a previous finding that OPNs pause during microsaccades (Brien et al., 2009) by quantifying the relationship between pause duration and microsaccade duration. Together, these findings further support a common saccadic burst generator for saccades and microsaccades (for review, see Hafed, 2011; Otero-Millan et al., 2011a).

\section{Evidence against Hering's law}

Over a century ago, Hering proposed the theory of "equal innervation" as a conceptual framework for studying binocular control. Hering's theory suggested that we are born with the ability to move our eyes in a coordinated fashion. Based on this theory, scientists predicted a distinct neural circuit for conjugate control (i.e., to move the eyes together). Specifically, it was proposed that the saccadic burst generator provides a conjugate premotor command to the eye muscles. Recent studies, however, have shown that SBNs do not strictly encode conjugate command signals but preferentially encode the movement of an individual eye (Fig. 4A) (Cullen and Van Horn, 2011). In this study, since many of the detected microsaccades were disconjugate, we similarly found that SBNs encode the movement of an individual eye during microsaccades. Accordingly, our results provide further evidence that the brainstem saccadic premotor pathway encodes integrated conjugate and vergence commands to control gaze in three-dimensional space. Notably, since SBNs receive excitatory connections from the SC, these results suggest that the SC should be reinvestigated to determine its involvement in generating saccades and microsaccades in three-dimensions (Fig. 4B).
These findings have important clinical implications. Abnormal frequency and size of microsaccades are characteristic of certain neurological diseases [e.g., progressive supranuclear palsy, Friedrich's ataxia (Troost and Daroff, 1977; Leigh and Zee, 2006; Fahey et al., 2008; Otero-Millan et al., 2011b)]. An analysis of the dynamic movement of each eye could be a valuable tool for establishing whether there is also a change in the proportion of disconjugate microsaccades in patients with these and other disorders, thereby providing an effective diagnostic marker.

\section{References}

Betta E, Turatto M (2006) Are you ready? I can tell by looking at your microsaccades. Neuroreport 17:1001-1004.

Brien DC, Corneil BD, Fecteau JH, Bell AH, Munoz DP (2009) The behavioural and neurophysiological modulation of microsaccades in monkeys. J Eye Mov Res 3:1-12.

Carpenter J, Bithell J (2000) Bootstrap confidence intervals: when, which, what? A practical guide for medical statisticians. Stat Med 19:1141-1164.

Cornsweet TN (1956) Determination of the stimuli for involuntary drifts and saccadic eye movements. J Opt Soc Am 46:987-993.

Cui J, Wilke M, Logothetis NK, Leopold DA, Liang H (2009) Visibility states modulate microsaccade rate and direction. Vision Res 49:228-236.

Cullen KE, Guitton D (1997) Analysis of primate IBN spike trains using system identification techniques. I. Relationship to eye movement dynamics during head-fixed saccades. J Neurophysiol 78:3259-3282.

Cullen KE, Van Horn MR (2011) The neural control of fast vs. slow vergence eye movements. Eur J Neurosci 33:2147-2154.

Cullen KE, Rey CG, Guitton D, Galiana HL (1996) The use of system identification techniques in the analysis of oculomotor burst neuron spike train dynamics. J Comput Neurosci 3:347-368.

Engbert R (2006) Microsaccades: a microcosm for research on oculomotor control, attention, and visual perception. Prog Brain Res 154:177-192.

Engbert R, Kliegl R (2003) Microsaccades uncover the orientation of covert attention. Vision Res 43:1035-1045.

Fahey MC, Cremer PD, Aw ST, Millist L, Todd MJ, White OB, Halmagyi M, Corben LA, Collins V, Churchyard AJ, Tan K, Kowal L, Delatycki MB (2008) Vestibular, saccadic and fixation abnormalities in genetically confirmed Friedreich ataxia. Brain 131:1035-1045.

Guerrasio L, Quinet J, Büttner U, Goffart L (2010) Fastigial oculomotor region and the control of foveation during fixation. J Neurophysiol 103:1988-2001.

Hafed ZM (2011) Mechanisms for generating and compensating for the smallest possible saccades. Eur J Neurosci 33:2101-2113.

Hafed ZM, Clark JJ (2002) Microsaccades as an overt measure of covert attention shifts. Vision Res 42:2533-2545.

Hafed ZM, Krauzlis RJ (2010) Microsaccadic suppression of visual bursts in the primate superior colliculus. J Neurosci 30:9542-9547.

Hafed ZM, Goffart L, Krauzlis RJ (2009) A neural mechanism for microsaccade generation in the primate superior colliculus. Science 323:940-943.

Hass CA, Horwitz GD (2011) Effects of microsaccades on contrast detection and $\mathrm{V} 1$ responses in macaques. J Vis 11:1-17.

Hays AV, Richmond BJ, Optican LM (1982) A UNIX-based multiple process system for real-time data acquisition and control. WESCON Conf Proc 2:1-10.

Hering E (1977) Lehre vom binokularen Sehen. [The theory of binocular vision (1868)]. New York: Plenum.

Herrington TM, Masse NY, Hachmeh KJ, Smith JE, Assad JA, Cook EP (2009) The effect of microsaccades on the correlation between neural activity and behavior in middle temporal, ventral intraparietal, and lateral intraparietal areas. J Neurosci 29:5793-5805.

Judge SJ, Richmond BJ, Chu FC (1980) Implantation of magnetic search coils for measurement of eye position: an improved method. Vision Res 20:535-538.

Kliegl R, Rolfs M, Laubrock J, Engbert R (2009) Microsaccadic modulation of response times in spatial attention tasks. Psychol Res 73:136-146.

Kowler E, Steinman RM (1979) Miniature saccades: eye movements that do not count. Vision Res 19:105-108.

Laubrock J, Engbert R, Kliegl R (2005) Microsaccade dynamics during covert attention. Vision Res 45:721-730.

Leigh RJ, Zee DS (2006) The neurology of eye movements. New York: Oxford UP. 
Martinez-Conde S (2006) Fixational eye movements in normal and pathological vision. Prog Brain Res 154:151-176.

Martinez-Conde S, Macknik SL, Hubel DH (2000) Microsaccadic eye movements and firing of single cells in the striate cortex of macaque monkeys. Nat Neurosci 3:251-258.

Martinez-Conde S, Macknik SL, Hubel DH (2002) The function of bursts of spikes during visual fixation in the awake primate lateral geniculate nucleus and primary visual cortex. Proc Natl Acad Sci U S A 99:13920-13925.

Martinez-Conde S, Macknik SL, Troncoso XG, Hubel DH (2009) Microsaccades: a neurophysiological analysis. Trends Neurosci 32:463-475.

Otero-Millan J, Troncoso XG, Macknik SL, Serrano-Pedraza I, MartinezConde S (2008) Saccades and microsaccades during visual fixation, exploration, and search: foundations for a common saccadic generator. J Vis 8:21.1-21.18.

Otero-Millan J, Macknik SL, Serra A, Leigh RJ, Martinez-Conde S (2011a) Triggering mechanisms in microsaccade and saccade generation: a novel proposal. Ann N Y Acad Sci 1233:107-116.

Otero-Millan J, Serra A, Leigh RJ, Troncoso XG, Macknik SL, MartinezConde S (2011b) Distinctive features of saccadic intrusions and microsaccades in progressive supranuclear palsy. J Neurosci 31:4379-4387.

Pastukhov A, Braun J (2010) Rare but precious: microsaccades are highly informative about attentional allocation. Vision Res 50:1173-1184.

Schwartz G (1978) Estimating the dimension of a model. Ann Stat 5:461-464.
Scudder CA, Kaneko CS, Fuchs AF (2002) The brainstem burst generator for saccadic eye movements: a modern synthesis. Exp Brain Res 142:439-462.

Snodderly DM, Kagan I, Gur M (2001) Selective activation of visual cortex neurons by fixational eye movements: implications for neural coding. Vis Neurosci 18:259-277.

Sokal RR, Rohlf FJ (1995) Biometry: the principles and practice of statistics in biological research. New York: Freeman.

Sylvestre PA, Cullen KE (2002) Dynamics of abducens nucleus neuron discharges during disjunctive saccades. J Neurophysiol 88:3452-3468.

Troost BT, Daroff RB (1977) The ocular motor defects in progressive supranuclear palsy. Ann Neurol 2:397-403.

Van Gisbergen JA, Robinson DA, Gielen S (1981) A quantitative analysis of generation of saccadic eye movements by burst neurons. J Neurophysiol 45:417-442.

Van Horn MR, Cullen KE (2008) Dynamic coding of vertical facilitated vergence by premotor saccadic burst neurons. J Neurophysiol 100:1967-1982.

Van Horn MR, Sylvestre PA, Cullen KE (2008) The brain stem saccadic burst generator encodes gaze in three-dimensional space. J Neurophysiol 99:2602-2616.

Walton MM, Mays LE (2003) Discharge of saccade-related superior colliculus neurons during saccades accompanied by vergence. J Neurophysiol 90:1124-1139. 